\title{
XPS/NEXAFS spectroscopic and conductance studies of glycine on AIGaN/GaN transistor devices
}

Matthew Myers ${ }^{c, d^{*}}$, Farah Liyana Muhammad Khir ${ }^{a, b}$, Michael A. Home ${ }^{a}$, Christopher Mennell ${ }^{a}$, Jeremy Gillbanks ${ }^{a}$, Anton Tadich ${ }^{\mathrm{e}}$, Murray V. Baker ${ }^{\mathrm{c}}$, Brett D. Nener ${ }^{\mathrm{a}}$, and Giacinta Parish $^{\mathrm{a}}$

\section{Affiliations}

aSchool of Electrical, Electronic and Computer Engineering, The University of Western Australia, 35 Stirling Hwy., Crawley, Western Australia 6009, Australia

E-mail: brett.nener@uwa.edu.au (B.D. Nener) and giacinta.parish@uwa.edu.au (G. Parish). ${ }^{\mathrm{b}}$ Faculty of Applied Sciences, Universiti Teknologi MARA, 40450 Shah Alam, Malaysia farah668@salam.uitm.edu.my (F.L.M. Khir)

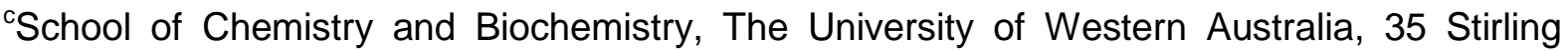
Hwy., Crawley, Western Australia 6009, Australia

E-mail: murray.baker@uwa.edu.au (M.V. Baker)

${ }^{d}$ CSIRO Energy, Kensington, Western Australia 6151, Australia

E-mail: Matt.Myers@csiro.au (M.Myers)

${ }^{\mathrm{e} A u s t r a l i a n}$ Synchrotron, 800 Blackburn Road, Clayton, Victoria 3168, Australia

\section{Keywords:}

NEXAFS, XPS, HEMT, sensor, zwitterion, transistor

\section{Abstract}

We report on a study using a combination of XPS/NEXAFS and conductivity measurements to develop a fundamental understanding of how dipolar molecules interact with the heterostructure device surface and affect the device conductivity of AIGaN/GaN heterostructure-based transistors. In such structures, which are increasingly being investigated for chemical and biological sensing, a 2-dimensional electron gas spontaneously forms at the layer interface that is sensitive to the charge characteristics of the exposed surface. Glycine, chosen for this study because it is the simplest of the amino acids and is known to form a zwitterionic configuration when stabilized through 
intermolecular interactions, was evaporated under ultra-high vacuum conditions onto the device surface and subsequently both XPS/NEXAFS and conductivity measurements were conducted. NEXAFS spectra show a preferential orientation for the glycine molecules on the surface and evidence for both neutral and zwitterionic species on the surface. In situ conductivity measurements suggest that the negatively charged carboxylate group is closest to the surface. These results are a unique and pivotal contribution to the previous and at times conflicting literature on the zwitterionic nature of glycine.

\section{Introduction}

Group III nitrides such as AIGaN and GaN exhibit a strong spontaneous and piezoelectric polarisation field [1] when grown as a wurtzite crystal structure. As a result, AIGaN/GaN hetero-structures can be utilised for high electron mobility transistors (HEMTs). AIGaN/GaN HEMTs are usually pursued for high power and/or high frequency electronics applications however they also show great promise as electrochemical sensors due to their excellent thermal and chemical stability, device compactness and high measurement sensitivity [2-4]. Through charge coupling, a 2-dimensional electron gas (2DEG) channel forms at the AIGaN/GaN interface and the conductivity of this channel is highly sensitive to surface charge characteristics, enabling its use for high performance ion selective field effect transistors (ISFETs). The presence of ions and/or dipoles on the surface induces an effective surface potential affecting the measured conductivity of the 2-DEG channel. We have recently shown that with appropriate PVC-based membranes these devices can be used to sense $\mathrm{Hg}^{2+}$ and $\mathrm{NO}_{3}{ }^{-}$ions in solution, with comparable or better performance when compared to existing portable ion sensor technologies $[5,6]$.

As part of our work using AIGaN/GaN transistors as a platform for sensing applications [7], we have undertaken a fundamental study on the relationship between the behaviour of surface molecular dipole species and device response. This relationship is important in the understanding of the inter-relationships between surface charge, surface potential and surface chemistry. The electrical potential due to the electrostatic interaction of the dipole layer on the surface with the 2DEG channel is given by: 


$$
\Delta V=N \mu(\cos \theta) / \varepsilon
$$

where $\Delta \mathrm{V}$ is the voltage change across a layer of thickness of one dipole, $\mathrm{N}$ is the surface density of dipole species, $\mu$ is the dipole moment, $\theta$ is the angle between the dipole and the surface normal and $\varepsilon$ is the absolute film permittivity. The first step in the study was to select a molecular dipole. For this work, glycine was selected since it is the simplest of the amino acids and is known to readily adopt a zwitterionic or dipolar characteristic on a variety of surfaces. It is well known that at $\mathrm{pH}$ values near 7 in solution, glycine exists as a zwitterion where the carboxyl group is de-protonated and the amine group is protonated with a neutral overall charge. However, in the gas phase, spectroscopic studies have shown that glycine is not a zwitterion, and this is consistent with ab initio calculations[8,9]. In the condensed phase either as a crystal or bound to a surface, the zwitterionic species is present presumably due to intermolecular interactions [10]. Based on this it is presumed that the zwitterionic species will exist on the GaN surface and exhibit a significant dipole moment which will affect the 2-DEG conductivity.

By systematically varying the incident angle during the spectroscopic experiment and modelling the changes to the spectra, NEXAFS provides an opportunity to determine the molecular orientation relative to the surface (i.e. it gives information regarding the angle $\theta$ in the electrostatic dipole equation) [11]. Numerous NEXAFS studies of glycine have been reported in the last twenty years; however, significant variation between the published spectra exists and may be partially attributed to differences in the sample preparation methods, as well as the natural tendency of glycine to exist in different molecular conformers and crystalline structures. Furthermore, very little has been published using NEXAFS to understand molecular orientation of dipoles on transistor surfaces and how the gate regions of transistors interact with these molecular dipoles. The results presented in this study are the first examples of NEXAFS spectra for glycine deposited on a highly polarised material, and (as will be demonstrated) do not directly resemble any spectra previously published. In order to explain these differences, and to leverage the current understanding of glycine 
spectra to maximum effect, a thorough analysis of the existing body of published work is also presented here.

The first published study of the NEXAFS spectrum of the glycine carbon K-edge is attributed to Boese et al.[12], who reported the spectra for a number of amino acid monomers as well as several polypeptides in 1997 . This paper focused on the spectral signatures of individual amino acids and evidence of the formation of peptide bonds, with the aim of using this information to predict and positively identify spectral features of complex proteins consisting of long peptide chains of such molecules. This theme was revisited in many later studies [1317] and extensive libraries of NEXAFS spectra are now available for both transmission [13] and partial electron yield [17] modes of measurement. A consensus amongst these works is the unambiguous assignment of the most prominent peak in the carbon K-edge spectrum, attributed to electron transitions from the innermost core level to the $\pi^{*}$ orbital associated with the carboxyl carbon, denoted $C 1 s \rightarrow \pi_{C=O}^{*}$.

Other early work included a report of the oxygen K-edge spectrum [18] and a series of two papers examining the absorption of glycine on copper substrates $[19,20]$. NEXAFS spectroscopy was employed, alongside a variety of other experimental and theoretical techniques, to deduce the nature of adsorption of glycine on copper substrates. These works identified three favourable chemisorption geometries of glycine on $\mathrm{Cu}$ (110) (see Figure 1).

Figure 1: Three of the most stable absorption geometries of glycine on $\mathrm{Cu}$ (110). The bridging geometry was found to be favoured at low coverages, the unidentate at higher coverages, and the flat-lying configuration predominates after annealing above $400 \mathrm{~K}$ [19].

NEXAFS spectra of glycine have also been the source of some controversy in the literature, namely the origin and assignment of some spectral features. There have been contradictory assignments of features associated with transitions from the carbon K-shell to anti-bonding orbitals of sigma symmetry. A comprehensive study conducted by Kaznacheyev et al. [13] attributed features in the $\sim 290-291 \mathrm{eV}$ range of transmission mode NEXAFS spectra of 
glycine to $\sigma_{C-N}^{*}$ transitions; in agreement with early theoretical results [21]. The same paper associated resonances around $294 \mathrm{eV}$ with $\sigma_{C-C}^{*}$ transitions, consistent with previously reported NEXAFS results for acetate [19] (a compound very similar to glycine, with a nearly identical carbon backbone). A later work by Zubavichus et al. disputed this assignment, attributing the $291 \mathrm{eV}$ feature instead to $\sigma_{C-C}^{*}$ transitions based on typical values observed in ISEELS spectra of gaseous ethane [15]. This extrapolation is less physically justifiable than the comparison with acetate as ethane does not feature the carboxyl group, common to both glycine and acetate, which imparts hybrid $\mathrm{sp}^{2}$ character $[19,20]$ to three of the orbitals centred around the constituent carbon atom. However later studies $[17,22]$ suggested that transitions to $\sigma_{C-N}^{*}$ orbitals are predominantly responsible for the lower energy peaks (near $291 \mathrm{eV}$ ), and $C 1 s \rightarrow \sigma_{C-C}^{*}$ transitions account for the latter higher energy peaks (near 294 $\mathrm{eV})$. This is a subtle but significant point as it has been noted by a more recent study that there is some degree of overlap of the contributions from both $\sigma_{C-N}^{*}$ and $\sigma_{C-C}^{*}$ transitions in the carbon spectrum, but nevertheless the resonance observed at $290 \mathrm{eV}$ is predominantly due to a $C 1 s \rightarrow \sigma_{C-N}^{*}$ transition [23].

More recent work has focused on spectral features associated with the different charge states of glycine (i.e. neutral or zwitterionic), especially those evident in $\mathrm{N} \mathrm{K}$-edge spectra. Messer et al. developed a novel measurement apparatus to record the first reported NEXAFS spectra of aqueous glycine [23] in transmission electron yield mode. This study examined the effects of protonation of the amine and carboxyl termini by recording NEXAFS spectra for solutions at different $\mathrm{pH}$ values. It also challenged the previously held conception that pre-edge features in the nitrogen ISEELS spectrum of gaseous glycine were due to solely to Rydberg resonances $[14,16]$ and corroborated this argument with experimental spectra and $a b$ initio calculations. A subsequent work by Otero and Urquhart [24] partially refuted this claim by demonstrating that structural differences in related amino acid salts (i.e. protonated vs. deprotonated amine groups) were related to the aforementioned pre-edge features, but also resulted in orbitals that possessed a Rydberg character that is noticeably 
quenched in the solid state. Another important contribution by Zubavichus et al. [22] further investigated the nature of pre-edge features in the $\mathrm{N} \mathrm{K}$-edge spectra through examination of several glycine-related salts and different crystalline polymorphs of glycine.

In this work NEXAFS/XPS spectroscopy combined with in situ conductivity measurements are used to develop an understanding of how dipoles and in particular these molecules interact with the AIGaN/GaN surface and consequently the 2DEG channel.

\section{Materials and Methods}

For these experiments, wafers were grown on sapphire substrates using metalorganic chemical vapour deposition at the University of California, Santa Barbara. The thickness of the AIGaN layer was $13.9 \mathrm{~nm}$ with an Al percentage of $24.3 \%$ and the bottom GaN layer had a thickness of approximate $2000 \mathrm{~nm}$. A top layer GaN cap of $2.4 \mathrm{~nm}$ was also grown to protect the electrical characteristics of the $\mathrm{AIGaN} / \mathrm{GaN}$ device. Carrier concentration and mobility were measured to be $7.2 \mathrm{e}^{2} \mathrm{~cm}^{-2}$ and $1555 \mathrm{~cm}^{2}(\mathrm{Vs})^{-1}$ respectively from Hall experiments. For these experiments two wafers were placed on the holder. One of these wafers served as a substrate for the XPS and NEXAFS experiments and the other wafer which contained processed sensor devices was used solely for conductivity measurements (based on a previous device design published elsewhere [6]).

Figure 2: Simplified diagram of the experimental apparatus in the measurement chamber. The ammeter connected to the reference wafer measures the replacement current that occurs due to the loss of electrons via the photoelectric effect; it therefore represents the total electron yield (TEY) for the NEXAFS experiments. Conversely the electron analyser records only a fraction of the emitted electrons; referred to as the partial electron yield (PEY). A negative voltage is applied to a metal grid (not shown) covering the aperture of the analyser, which precludes measurement of lower energy electrons 
The soft X-ray (SXR) beam-line at the Australian synchrotron facility in Melbourne was utilized for the XPS/NEXAFS measurements in combination with in situ glycine deposition and subsequent conductivity measurements. Here, horizontally polarized soft x-ray radiation is produced from an undulator light source. The beamline, based around a plane diffraction grating monochromator (line spacing of 1200 lines $/ \mathrm{mm}$ ) is used to collimate and monochromatize the X-ray beam. The base pressure in the system was approximately $5 \times 10^{-10}$ Torr. XPS survey scans were undertaken at a photoelectron energy of $700 \mathrm{eV}$. The C $K$ edge NEXAFS data ( $h v=280-320 \mathrm{eV}, 0.1 \mathrm{eV}$ step size) were acquired for this study. Two different, simultaneous, detection modes were used to measure the x-ray absorption signal: surface sensitive Auger electron yield (AEY), obtained by monitoring the sample drain current, and the more bulk sensitive total fluorescence yield (TFY), achieved by using a retarding grid detector directed toward the sample and located $30^{\circ}$ above the incoming beam. The NEXAFS spectra were normalized with respect to the incidental photon flux by dividing the sample signal $I_{s}$ with the incident photon flux $I_{0}$, monitored using a $50 \%$ transmissive gold grid located just before the sample. A linear background was subtracted from the spectra by fitting the pre-edge region. The approximate energy resolution of the NEXAFS spectra is around $0.1 \mathrm{eV}$.

Figure 2 illustrates the sample configuration for this study with separate wafers for X-ray analysis and in situ conductivity measurements. Deposition of the molecules onto both was achieved via thermal evaporation of a source in the sample preparation chamber of the beamline. It is important to note that no metal gate was attached to the devices. Quartz crystal microbalance (QCM) measurements were used to monitor the rate of evaporation and deposition of molecular membranes onto the semiconductor wafers. Additionally, an in situ mass spectrometer was used to confirm the source evaporation.

Cleaning of the samples prior to measurement was performed with isopropyl alcohol and drying by nitrogen gas, and then, after loading into the chamber, by sputtering the surface with argon gas for 90 minutes at a potential of $0.5 \mathrm{kV}$. For deposition, glycine was heated initially to $152^{\circ} \mathrm{C}$ and cooled to $147^{\circ} \mathrm{C}$ for thermal evaporation. QCM measurements 
determined the rate of deposition to be $0.25-0.3 \AA / s$. The total cumulative time of glycine deposition was 11 minutes (one 1 minute deposition followed by five 2 minute depositions). Before and after each deposition, conductance measurements and XPS measurement were conducted. For the final deposition, NEXAFS measurement was also undertaken. For XPS and NEXAFS, the incident beamline was directed on the reference structure surface. As a precaution, radiation damage to the adhered glycine was minimised by slowly scanning the region of the sample onto which the beamline was directed for each scan [25]. Conductivity measurements were obtained from the processed sensor.

The data processing of the spectra was accomplished using an Igor Pro software package (Australian Synchrotron NEXAFS Loader v1.28) developed by Dr Eliot Gann of the Australian Synchrotron. In this software package, a double normalisation procedure was used to correct the NEXAFS spectra [26]. In the first normalisation the true flux profile is corrected by division using a photodiode. In the second normalisation, a reference foil signal from highly pyrolytic graphite with a distinct resonance at $291.65 \mathrm{eV}$ is used to translate the energy scale of each spectra. This second step is used due to carbon contamination that is widely found in NEXAFS beamlines. For this data normalization and peak fitting, a gold grid with a characteristic binding energy of $84 \mathrm{eV}$ and adventitious carbon with a resonance at $284.6 \mathrm{eV}$ were used as the reference for absolute energy scale calibration. NEXAFS spectra measurements of the samples were taken with the following photon polarizations with respect to the device surface: $20^{\circ}, 40^{\circ}, 55^{\circ}, 70^{\circ}$ and $90^{\circ}$.

\section{Results and Discussion}

\section{NEXAFS spectroscopy of glycine evaporated on a Si wafer}

For baseline purposes, the carbon K-edge spectra was acquired for glycine on a silicon surface with a native oxide as is typically present on silicon wafers, and is shown in Figure 3. The spectrum closely matches that reported for a $55^{\circ}$ scan of solid glycine on indium foil [22], in which a thick deposition was positively identified as single-phase $\alpha$-glycine via X-ray diffraction techniques. This conclusion is unsurprising as it is well known that $\alpha$-glycine is the 
most stable crystalline polymorph under most conditions [27]. On the basis of this and the close similarity between our results and those presented in [22], it is concluded that the adsorbed glycine on silicon substrate is predominantly zwitterionic glycine, likely the alpha polymorph.

Figure 3: Electron yield NEXAFS C K-edge spectrum recorded for glycine evaporated onto a native oxide Si wafer, with peak assignments shown.

The location of all main spectral features in Figure 3 are consistent with previously reported NEXAFS spectra of solid glycine. Consequently, the most prominent peaks have been assigned based on past studies. The sharp peak centred at approximately $288.6 \mathrm{eV}$ is unambiguously attributed to $C 1 s \rightarrow \pi_{(C=O)}^{*}$ transitions [13]. The $\sigma^{*}$ resonances are assigned to the following final state orbitals:

- $\quad 291 \mathrm{eV}$ to $\sigma_{\mathrm{C}-\mathrm{NH}_{3}^{+}}^{*}$

- $\quad 294.2 \mathrm{eV}$ to $\sigma_{C-C}^{*}$

- $\quad 300.7 \mathrm{eV}$ to $\sigma_{C-O O^{-}}^{*}$

There is an additional peak around $313 \mathrm{eV}$ which is not evident in previously published spectra of glycine and could possibly be attributed to transitions from the carbon atoms in the first monolayer of chemisorbed glycine at the glycine/oxide interface, which would exist in a distinctly different local atomic environment to the surface layers typically studied.

\section{XPS and NEXAFS spectroscopy of glycine evaporated on a GaN-capped AIGaN/GaN wafer}

For glycine evaporated onto GaN-capped AlGaN/GaN substrates, XPS scans were conducted on the substrates after evaporation of glycine. XPS of a bare GaN surface has a binding energy peak at 393-395 eV associated with $\mathrm{N}$ and another peak at $284.6 \mathrm{eV}$ associated with adventitious carbon [28]. As seen in Figure 4a, the N peak shifted to $404 \mathrm{eV}$ which corresponds to the $-\mathrm{NH}_{2}$ peak. Furthermore, Figure $4 \mathrm{~b}$ shows two new peaks at 290 $\mathrm{eV}$ and $291 \mathrm{eV}$ corresponding to the methylene and carboxylate $\mathrm{C}$ atoms in glycine, respectively. The $\mathrm{O}$ peak in Figure 4c has two features at approximately $535 \mathrm{eV}$ and $538 \mathrm{eV}$ while the native oxide of GaN has a peak at $533 \mathrm{eV}$. The peak at $535 \mathrm{eV}$ is significantly 
larger in intensity compared to the peak at $538 \mathrm{eV}$. The higher energy peak at $535 \mathrm{eV}$ is assigned to carbonyl oxygen while the peak at $538 \mathrm{eV}$ is assigned to a hydroxyl oxygen [29] suggesting that the zwitterionic species is dominant.

Figure 4: XPS spectra of $\mathrm{N} 1 \mathrm{~s}(\mathrm{a}), \mathrm{C}$ 1s (b) and $\mathrm{O}$ 1s (c) peaks after glycine evaporation for a total of 11 minutes

Following the final deposition and XPS scan, a full series of carbon K-edge NEXAFS experiments at the five different angles was conducted. The carbon K-edge spectra is important as the carbon atoms in glycine are participant in all bonds involving heavier atoms within the molecule; these bonds correspond to the most reliable and readily interpreted NEXAFS features [11]. The $55^{\circ}$ scan is the least sensitive to molecular orientation and related linear dichroism effects [11] and its spectrum is shown in Figure 5.

Figure 5: TEY C K-edge spectrum for glycine evaporated onto a GaN-capped AIGaN/GaN wafer, for $55^{\circ}$ scan angle. (Add something in caption about A-F depending on what you decide in relation to my comment.)

A broad pre-edge peak labelled A is observed at approximately 283 to $285 \mathrm{eV}$ and the edge is at $288.4 \mathrm{eV}$. The sharp peak, labelled $\mathrm{B}$ in Figure 5 , can again be ascribed to a $C 1 \mathrm{~s} \rightarrow$ $\pi_{C=O}^{*}$ transition [13] and is located at approximately $288.65 \mathrm{eV}$ (determined by fitting the spectra to a series of Gaussian peaks). For the remaining $\sigma^{*}$ (i.e. C, D, E and F) peak resonances, peak assignment is not as straightforward. Peaks C and D are at 289.95 and $290.75 \mathrm{eV}$, respectively. Peak C is interesting as it has not been observed in past studies of solid glycine (nor in the spectrum of glycine deposited on a Si wafer shown in Figure 3); therefore the 'building block' principle is employed to assign this spectral feature based on similar molecules. The carbon K-edge spectrum of solid $\mathrm{Na}^{+} \mathrm{Gly}^{-}$has a peak at $289.9 \mathrm{eV}$ of near-identical width [22], and a similar feature c.a. $289.9 \mathrm{eV}$ has been found in the spectrum of lysine and attributed to $\mathrm{C} 1 s \rightarrow \sigma_{C-N H_{2}}^{*}$ transitions [17]. It is plausible then that this feature is due to transitions to a neutral $\sigma_{C-N H 2}^{*}$ orbital. Conversely, the location of peak $D$ at $290.75 \mathrm{eV}$ suggests it corresponds to $\sigma_{C-N H_{3}^{+}}^{*}$ transitions $[13,22]$, though it is significantly narrower than those previously reported. This concurrent assignment of features with both the zwitterionic $\sigma_{C-N H 2}^{*}$ and neutral $\left(\sigma_{C-N H_{3}^{+}}^{*}\right)$ species is problematic as an accurate assignment 
of spectral features presumes that the peak assignments are consistent. Peak $E$ is located at approximately $295 \mathrm{eV}$ and is attributed to $\sigma_{C-C}^{*}$ transitions; it is shifted $+0.8 \mathrm{eV}$ from the position of the associated feature in glycine on Si spectrum, and that reported for a-glycine [22]. It is also considerably broadened in width, and reduced in amplitude. The last remaining $\sigma^{*}$ feature, peak $\mathrm{F}$ in 5 , is attributed to a superposition of $C 1 s \rightarrow \sigma_{C=O}^{*}, C 1 s \rightarrow$ $\sigma_{C-O H}^{*}$, and $C 1 s \rightarrow \sigma_{C-O O^{-}}^{*}$ transitions.

In the case of zwitterionic glycine, only one $\sigma^{*}$ peak is expected because the two oxygen atoms attached to the carboxyl carbon have near-identical bonding configurations due to bond resonance $[13,22]$. The local electron configuration of each oxygen atom is identical in the zwitterionic molecule, and the resulting bond lengths are very similar. Consequently, transitions to either $\sigma_{C-O o^{-}}^{*}$ orbital are superimposed at approximately $300.4 \mathrm{eV}[13]$. However, for neutral glycine, the electron configurations are completely different and consequently the bond lengths are also markedly different.

Unfortunately, there is no existing NEXAFS data for neutral glycine in the solid state. However, an early study conducted by Kaznacheyev et al. [13] examined the spectrum of solid glycinium, $\mathrm{H}_{3} \mathrm{~N}-\mathrm{CH}_{2}-\mathrm{COOH}^{+}$, the cationic conjugate acid of glycine. This was achieved by drying a highly acidic $\left(\mathrm{pH}\right.$ 1.2) aqueous solution onto a $\mathrm{Si}_{3} \mathrm{~N}_{4}$ substrate. Since the carboxyl group of glycinium is protonated in the solid state, the local atomic environment around the carboxyl carbon is very similar to that of neutral glycine, so it is reasonable to expect neutral glycine to exhibit a similar feature to the faintly visible $C 1 s \rightarrow \sigma_{C=O}^{*}$ resonance identified at c.a. $302 \mathrm{eV}$ [13] in that study. Similar reasoning was employed in the previous assignment of the $\sigma_{\mathrm{C}-\mathrm{NH}_{2}}^{*}$ feature. Unfortunately, no feature corresponding to $\mathrm{C} 1 \mathrm{~s} \rightarrow \sigma_{\mathrm{C}-\mathrm{OH}}^{*}$ transitions is discernible in the aforementioned $\mathrm{Na}^{+}$Gly. spectrum [13]. Such a feature is expected to be weaker because the longer $\mathrm{C}-\mathrm{OH}$ bond will reduce the amount of overlap between the individual atomic orbitals involved in its formation. A further complication that arises is the presence of Rydberg states that are believed to contribute significantly to the overall density of states in this region (rationalised by ab initio calculations in [23]). For the 
aforementioned reasons, quantitative interpretation of features above approximately $296 \mathrm{eV}$ is likely to be very error-prone, and this region will therefore be treated relatively qualitatively in subsequent analysis.

These results contradict the broadly accepted view that glycine exists almost exclusively in zwitterionic form in the solid state [13, 14, 27]. However, theoretical calculations have shown that the neutral state is dominant in the gas phase and if not stabilized through hydrogen bonding can exist in the neutral form on a surface $[30,31]$. It is possible then to presume that the first layer of glycine on the surface exists in the neutral form while the upper layers exist in the zwitterionic form and this would be consistent with the spectrum obtained on the GaN surface.

This is not the first time that the conception of a predominantly zwitterionic species has been challenged. As discussed in the introduction one previous study of glycine and related oligopeptides by Zubavichus et al [15] suggested that shifts in the shape of the main $\pi_{C=O}^{*}$ resonance, and the appearance of pre-edge features in the $\mathrm{N} \mathrm{K}$-edge, could reflect changes in the neutral:zwitterionic ratio. This view has been contested [14], and subsequent studies have demonstrated that the aforementioned pre-edge features (just before the nitrogen $\mathrm{K}$ edge) are directly related to the formation of peptide bonds [16] in the solid form (although in the gas phase similar features manifest in neutral glycine spectra [32]). Similarly, shifts of approximately $0.3 \mathrm{eV}$ in the position of $C 1 s \rightarrow \pi_{C=O}^{*}$ resonances have been replicated in several studies examining proteins with peptide bonds [14, 16, 33]. However, this effect is not singularly related to peptide bond formation as demonstrated in a landmark study of aqueous glycine [23]. In this work, protonation of the amine and carboxyl groups was controlled via $\mathrm{pH}$ and transitions from both the $\mathrm{C} 1 \mathrm{~s}$ and $\mathrm{O} 1 \mathrm{~s}$ energy levels to the $\pi^{*}$ orbitals of the carboxyl group were found to undergo significant red-shifts of 0.15 and $0.25 \mathrm{eV}$ respectively.

If the zwitterionic and neutral species are both present and possess different molecular orientations, there should be some evidence of angular variation in the $\pi_{C=O}^{*}$ resonances. 
Specifically, a noticeable shift in the resonance position could imply protonation of the carboxyl group in line with the results reported by Besser et al in 2005 [23]. If the abovementioned effect is present, it can only be unambiguously attributed to protonation of the carboxyl group in the absence of peptide bonds, for which a similar energetic shift has been demonstrated $[14,16]$.

Figure 6: Angular variation between the spectra recorded at $20^{\circ}$ and $90^{\circ}$ incidence for $\mathrm{GaN}$ capped $\mathrm{AIGaN} / \mathrm{GaN}$ with 11 minutes of glycine deposition. The top graph shows both regular spectra, the bottom is the difference spectrum.

Figure 6 displays the spectra recorded for this study at incidence angles of $20^{\circ}$ and $90^{\circ}$, as well as their difference spectrum. The difference spectrum highlights the intensity variation between the two spectra: two strong 'spikes' located at approximately 288.4 and $288.9 \mathrm{eV}$ indicate that the main $\pi_{C=O}^{*}$ peak has undergone a slight shift in energy between the two scans. This is faintly visible in the overlaid individual spectra in Figure 6. Another two distinct features in the difference spectrum appear at 289.9 and $290.9 \mathrm{eV}$; corresponding to intensity variations in the features assigned to $\sigma_{C-N H_{2}}^{*}$ and $\sigma_{C-N H_{3}^{+}}^{*}$ transitions. The opposing sign in the difference signal associated with these two features is due to their reversed movement as the incidence angle is varied: the $\sigma_{C-N H 2}^{*}$ peak exhibits a marked increase in intensity as the sample is brought orthogonal to the incoming radiation, whereas the $\sigma_{C-N H_{3}^{+}}^{*}$ peak decreases significantly. This suggests the corresponding bonds have distinctly different orientations. There is some evidence of linear dichroism at higher energies but it is less pronounced than the lower energy variations. If significant quantities of both zwitterionic and neutral species are present in the sample, then the region past $296 \mathrm{eV}$ is also expected to contain a superposition of at least three resonant features associated with the different $\sigma^{*}$ transitions in the carboxyl group. This could explain the low intensity, broad peak in the difference spectrum at approximately $305 \mathrm{eV}$, which cannot be numerically reproduced by the single $300.4 \mathrm{eV} \sigma_{\mathrm{C}-\mathrm{oo}}^{*}[13]$ resonance feature associated with $100 \%$ zwitterionic glycine. The results presented in Figure 6 are significant in the context of the literature. They are distinctly different to those presented in an angle-resolved NEXAFS study of the pristine $\alpha-$ 
glycine polymorph [22]. The reported a-glycine difference spectrum exhibits no evidence of an energetic shift in the $\mathrm{C} 1 \mathrm{~s} \rightarrow \pi_{C=O}^{*}$ resonance, in contrast to the data presented here. The distinct features present here and ascribed to transitions to $\sigma_{C-N H_{2}}^{*}$ and $\sigma_{C-N H_{3}^{+}}^{*}$ orbitals are also absent in the published spectra for $\alpha$-glycine. This provides further compelling evidence that the glycine probed in the NEXAFS experiments here is not $\alpha$-glycine, and strongly corroborates the assertion that both neutral and zwitterionic species are present in comparable quantities in the solid state.

Other authors, most notably Zubavichus et al., have speculated that such a phenomenon of types of species coexisting is likely [22], but the experimental evidence presented in their past studies has been inconclusive [15]. They observed a similar shift in the main $C 1 s \rightarrow$ $\pi_{C=O}^{*}$ resonance to that reported here [15], and attributed this shift to evidence of the neutral species with an $\mathrm{NH}_{2}$ group. However, they also attributed the emergence of a strong preedge feature in the nitrogen spectrum, to evidence of neutral species whereas no such preedge feature is evident in the spectra reported here. On the other hand, the spectra in this work contain many indicators of the neutral species (as discussed earlier) not evident in [15]. This suggests that unintentional formation of peptide bonds during sample preparation could explain the effects ascribed to neutral species by Zubavichus et al. in [15], which is shared by other authors [14]. It is likely then, that this study may be the first to positively identify appreciable quantities of both species in the solid-phase through NEXAFS spectroscopy.

\section{Conductivity measurements}

Prior to glycine adsorption, the potential across the sensor was $158.7 \mathrm{mV}$. As previously described, the 11 minutes total deposition of glycine consisted of one deposition of 1 minute duration and five depositions of 2 minute duration. The device conductivity remained relatively constant after the first deposition, with the mean potential across all readings being $160 \mathrm{mV}$. For the five depositions of 2 minute duration, there was a distinct response to each deposition with a mean $\Delta \mathrm{V}$ (voltage step change from before and after each deposition) of $1.5 \mathrm{mV}$. Control experiments with no deposition showed no detectable change in the device 
response. An increase in the voltage implies that the device is becoming less conductive and is consistent with the carboxylate group being closest to the surface, reducing the concentration of electrons in the 2-DEG channel. Unfortunately, the cumulative effect of the combined depositions on device conductivity could not be measured. As previously discussed, the glycine deposition and conductivity measurements were both performed in the sample preparation chamber, allowing the immediate effect of deposition to be assessed. However, the stage containing both samples was moved from the sample preparation chamber to the analysis chamber for each XPS/NEXAFS measurement. Unfortunately the conductivity is affected by light [34] and due to light exposure when the sample was moved between chambers the baseline conductivity therefore shifted after each measurement. This effect persists for an extended period of time making a determination of the cumulative effect due to deposition difficult.

\section{Conclusion}

XPS and NEXAFS measurements of glycine evaporated onto Si wafers and GaN-capped AIGaN/GaN wafers were conducted. Comparison of both similarities and differences between published XPS and NEXAFS spectra for various forms of glycine on different surfaces supports the existence of both the neutral and zwitterionic species on the surface. Furthermore, angular dependant NEXAFS reveals that the neutral and zwitterionic species have different orientations on the surface, pointing to the possibility of a layered structure. In addition, the conductance measurements indicate that the negatively charged end of the zwitterionic species is closest to the surface. In situ conductivity measurements further corroborate the presence of negative charge close to the surface as a result of the depositions. This is the first study that integrates between XPS, NEXAFS and conductivity measurement in situ to monitor both surface and electrical behaviour of deposited molecular species. As well as providing insight into molecular interactions at the surface of GaN-based sensor structures, the intriguing results add a new dimension, and even some clarity, to the previous and at times conflicting literature on the zwitterionic nature of glycine.

Acknowledgements 
We would like to acknowledge the Mishra group at the University of California Santa Barbara for the AIGaN/GaN samples used in this study. This work was performed in part at the Western Australian Node of the Australian National Fabrication Facility, a company established under the National Collaborative Research Infrastructure Strategy to provide nano- and micro-fabrication facilities for Australia's researchers. The research was undertaken at the Australian Synchrotron, Victoria, Australia with project grants of AS123/SXRFI/5253/Surface Chemistry of AIGaN \& GaN Surfaces for Sensor Applications and AS123/SXRFI/8820/Fundamental investigations of dipolar molecule interactions with AIGaN/GaN HEMT devices using NEXAFS and in situ conductivity measurements. We would also like to thank Bruce Cowie at the Australian Synchrotron for his help with XPS experiments. FLMK was funded by a PhD scholarship from the Government of Malaysia.

\section{References}

[1] J.P. Ibbetson, P.T. Fini, K.D. Ness, S.P. DenBaars, J.S. Speck, U.K. Mishra, Polarization effects, surface states, and the source of electrons in AIGaN/GaN heterostructure field effect transistors, Applied Physics Letters, 77(2000) 250-2.

[2] G. Steinhoff, O. Purrucker, M. Tanaka, M. Stutzmann, M. Eickhoff, AlxGa1-xN - A new material system for biosensors, Advanced Functional Materials, 13(2003) 841-6.

[3] A.B. Encabo, J. Howgate, M. Stutzmann, M. Eickhoff, M.A. Sánchez-García, Ultrathin GaN/AIN/GaN solution-gate field effect transistor with enhanced resolution at low source-gate voltage, Sensors and Actuators B: Chemical, 142(2009) 304-7.

[4] M. Eickhoff, J. Schalwig, G. Steinhoff, O. Weidemann, L. Gorgens, R. Neuberger, et al., Electronics and sensors based on pyroelectric AlGaN/GaN heterostructures - Part B: Sensor applications, Group lii-Nitrides and Their Heterostructures: Growth, Characterization and Applications, (2003) 1908-18. [5] M. Asadnia, M. Myers, N.D. Akhavan, K. O'Donnell, G.A. Umana-Membreno, U.K. Mishra, et al., Mercury(II) selective sensors based on AIGaN/GaN transistors, Analytica Chimica Acta, 943(2016) 17.

[6] M. Myers, F.L.M. Khir, A. Podolska, G.A. Umana-Membreno, B. Nener, M. Baker, et al., Nitrate ion detection using AlGaN/GaN heterostructure-based devices without a reference electrode, Sensors and Actuators B-Chemical, 181(2013) 301-5.

[7] A. Podolska, L.C. Hool, K.D.G. Pfleger, U.K. Mishra, G. Parish, B.D. Nener, AlGaN/GaN-based biosensor for label-free detection of biological activity, Sensors and Actuators B-Chemical, 177(2013) 577-82.

[8] R.D. Suenram, F.J. Lovas, Millimeter wave spectrum of glycine, Journal of Molecular Spectroscopy, 72(1978) 372-82.

[9] M.S. Gordon, J.H. Jensen, Understanding the Hydrogen Bond Using Quantum Chemistry, Accounts of Chemical Research, 29(1996) 536-43.

[10] Y. Zubavichus, A. Shaporenko, M. Grunze, M. Zharnikov, Solid-State Near-Edge X-ray Absorption Fine Structure Spectra of Glycine in Various Charge States, The Journal of Physical Chemistry B, 110(2006) 3420-7.

[11] J. Stohr, NEXAFS Spectroscopy, Berlin: Springer; 1992.

[12] J. Boese, A. Osanna, C. Jacobsen, J. Kirz, Carbon edge XANES spectroscopy of amino acids and peptides, Journal of Electron Spectroscopy and Related Phenomena, 85(1997) 9-15. 
[13] K. Kaznacheyev, A. Osanna, C. Jacobsen, O. Plashkevych, O. Vahtras, H. Agren, et al., Innershell absorption spectroscopy of amino acids, Journal of Physical Chemistry A, 106(2002) 3153-68.

[14] M. Gordon, G. Cooper, C. Morin, T. Araki, C. Turci, K. Kaznatcheev, et al., Inner-Shell Excitation Spectroscopy of the Peptide Bond: Comparison of the C 1s, N 1s, and O 1s Spectra of Glycine, GlycylGlycine, and Glycyl-Glycyl-Glycine, Journal of Physical Chemistry A, 107(2003) 6144-59.

[15] Y. Zubavichus, M. Zharnikov, A. Schaporenko, M. Grunze, NEXAFS study of glycine and glycinebased oligopeptides, Journal of Electron Spectroscopy and Related Phenomena, 134(2004) 25-33.

[16] G. Cooper, M. Gordon, D. Tulumello, C. Turci, K. Kaznatcheev, A. Hitchcock, Inner shell excitation of glycine, glycyl-glycine, alanine, and phenylalanine, Journal of Electron Spectroscopy and Related Phenomena, 137-140(2004) 795-9.

[17] Y. Zubavichus, A. Schaporenko, M. Grunze, M. Zharnikov, Innershell absorption spectroscopy of amino acids at all relevant absorption edges, Journal of Physical Chemistry A,, 109(2005) 6998-7000.

[18] M. Tanaka, K. Nakagawa, T. Koketsu, A. Agui, A. Yokoya, Oxygen K-edge X-ray absorption near edge structure (XANES) of sublimated films of amino acids, Journal of Synchrotron Radiation, 8(2001) 1009-11.

[19] J. Hasselstrom, O. Karis, M. Weinelt, N. Wassdahl, A. Nilsson, M. Nyberg, et al., The adsorption structure of glycine adsorbed on $\mathrm{Cu}(110)$; comparison with formate and acetate/ $\mathrm{Cu}(110)$, Surface Science, 407(1998) 221-36.

[20] M. Nyberg, J. Hasselstrom, O. Karis, N. Wassdahl, M. Weinelt, A. Nilsson, et al., The electronic structure and surface chemistry of glycine adsorbed on $\mathrm{Cu}(110)$, Journal of Chemical Physics, 112(2000) 5420-7.

[21] V. Carravetta, O. Plashkevych, H. Agren, A theoretical study of the near-edge x-ray absorption spectra of some larger amino acids, Journal of Chemical Physics, 109(1998) 1456-64.

[22] Y. Zubavichus, A. Shaporenko, M. Grunze, M. Zharnikov, Solid-state near-edge x-ray absorption fine structure spectra of glycine in various charge states, Journal of Physical Chemistry B, 110(2006) 3420-7.

[23] B. Messer, C. Cappa, J. Smith, K. Wilson, M. Gilles, R. Cohen, et al., pH dependence of the electronic structure of glycine, Journal of Physical Chemistry B, 109(2005) 5375-82.

[24] E. Otero, S.G. Urquhart, \Nitrogen 1s near-edge x-ray absorption fine structure spectroscopy of amino acids: Resolving zwitterionic effects, Journal of Physical Chemistry A, 110(2006) 12121-8.

[25] Y. Zubavichus, M. Zharnikov, A. Schaporenko, M. Grunze, NEXAFS study of glycine and glycinebased oligopeptides, Journal of Electron Spectroscopy and Related Phenomena, 134(2004) 25-33.

[26] J. Stöhr, NEXAFS Spectroscopy: Springer; 1992.

[27] C. Schwartz, R. Saykally, D. Prendergast, An analysis of the NEXAFS spectra of a molecular crystal: alpha-glycine, Journal of Chemical Physics, 133(2010).

[28] F.L.M. Khir, M. Myers, A. Podolska, T.M. Sanders, M. Baker, B.D. Nener, et al., Synchrotronbased XPS studies of AIGaN and GaN surface chemistry, Applied Surface Science, 314(2014) 850-7. [29] A.R. Slaughter, M.S. Banna, Core-photoelectron binding energies of gaseous glycine: correlation with its proton affinity and gas-phase acidity, The Journal of Physical Chemistry, 92(1988) 2165-7. [30] A. Rimola, B. Civalleri, P. Ugliengo, Neutral vs Zwitterionic Glycine Forms at the Water/Silica Interface: Structure, Energies, and Vibrational Features from B3LYP Periodic Simulations, Langmuir, 24(2008) 14027-34.

[31] A. Rimola, M. Sodupe, S. Tosoni, B. Civalleri, P. Ugliengo, Interaction of Glycine with Isolated Hydroxyl Groups at the Silica Surface: First Principles B3LYP Periodic Simulation, Langmuir, 22(2006) 6593-604.

[32] O. Plekan, V. Feyerb, R. Richter, M. Coreno, M. DeSimone, K. Prince, et al., An X-ray absorption study of glycine, methionine and proline, Journal of Electron Spectroscopy and Related Phenomena, 115(2007) 47-53.

[33] Y. Zubavichus, A. Shaporenko, M. Grunze, M. Zharnikov, NEXAFS spectroscopy of biological molecules: From amino acids to functional proteins, Nuclear Instruments and Methods in Physics Research A, 603(2009) 111-4. 
[34] M. Eckshtain-Levi, E. Capua, Y. Paltiel, R. Naaman, Hybrid Sensor Based on AIGaN/GaN Molecular Controlled Device, ACS Sensors, 1(2016) 185-9. 
Click here to download high resolution image

\section{Bridging}

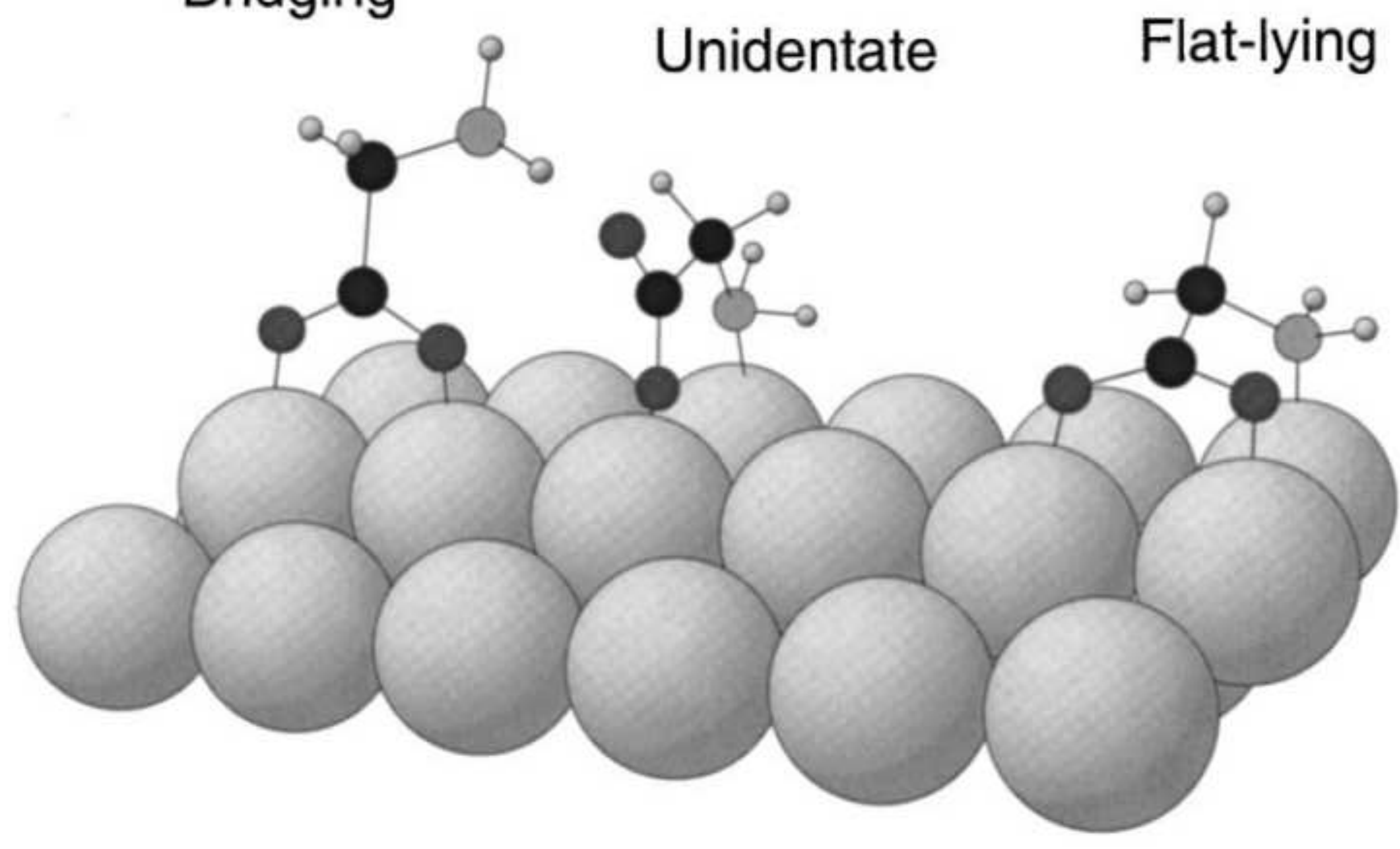




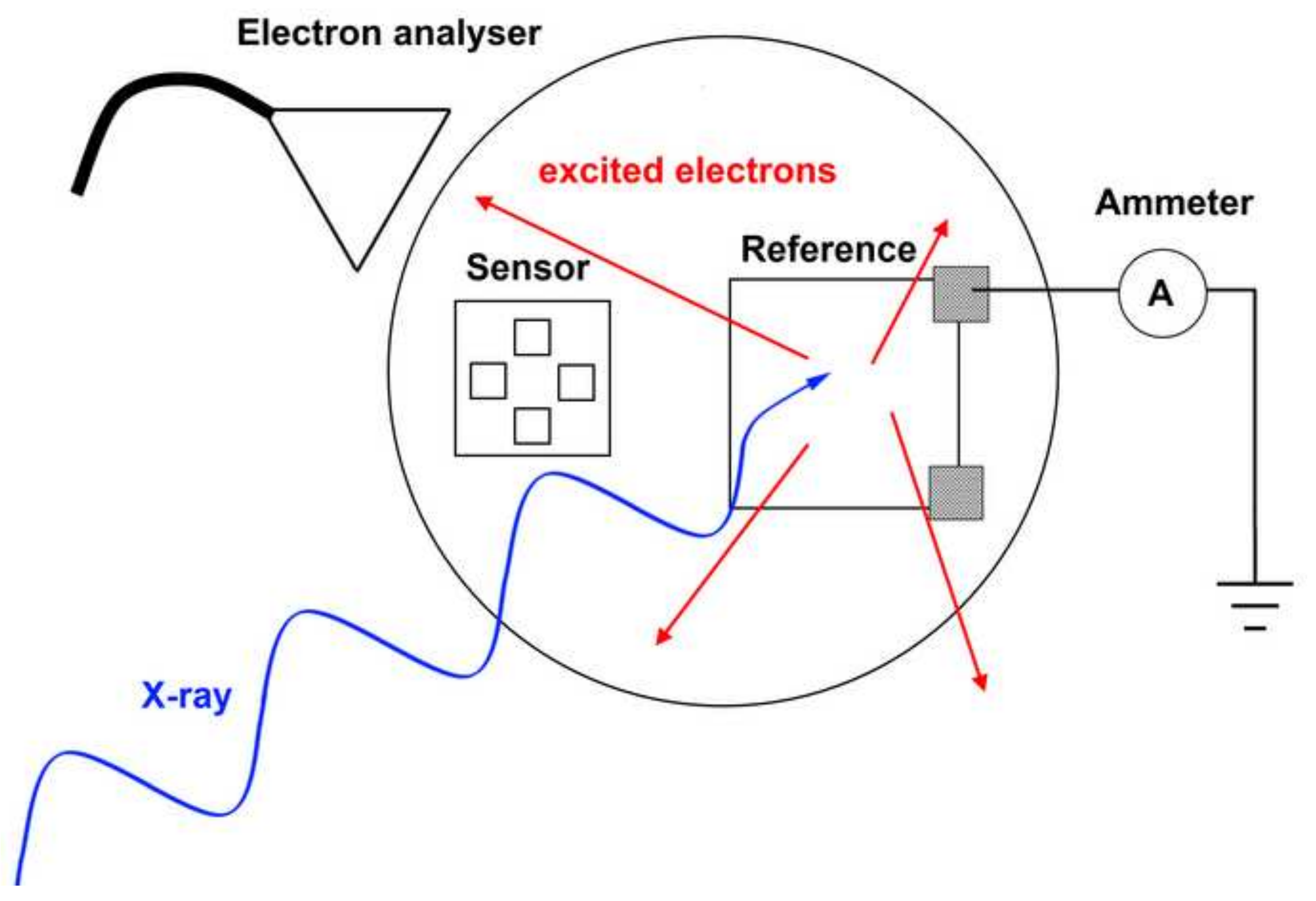




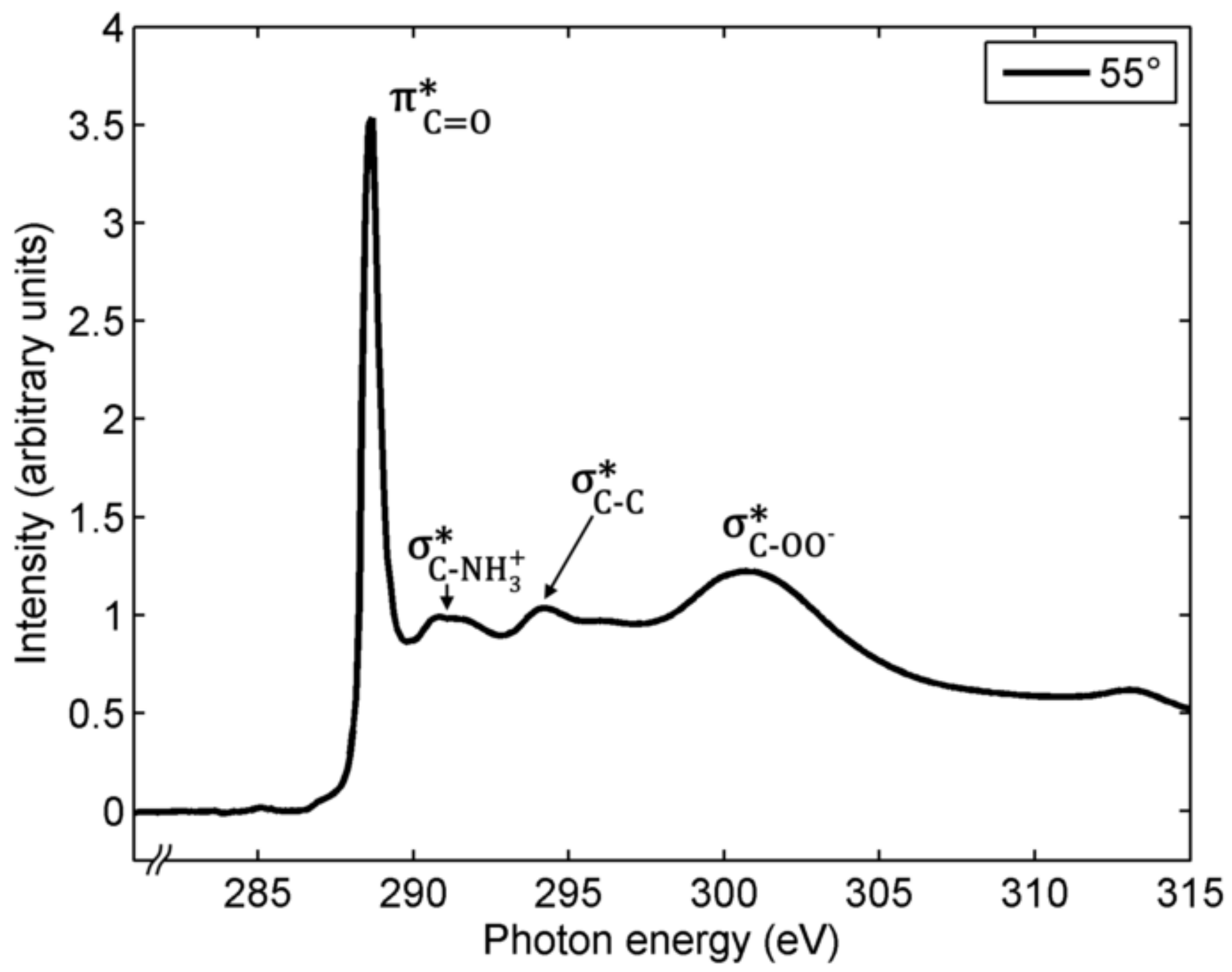




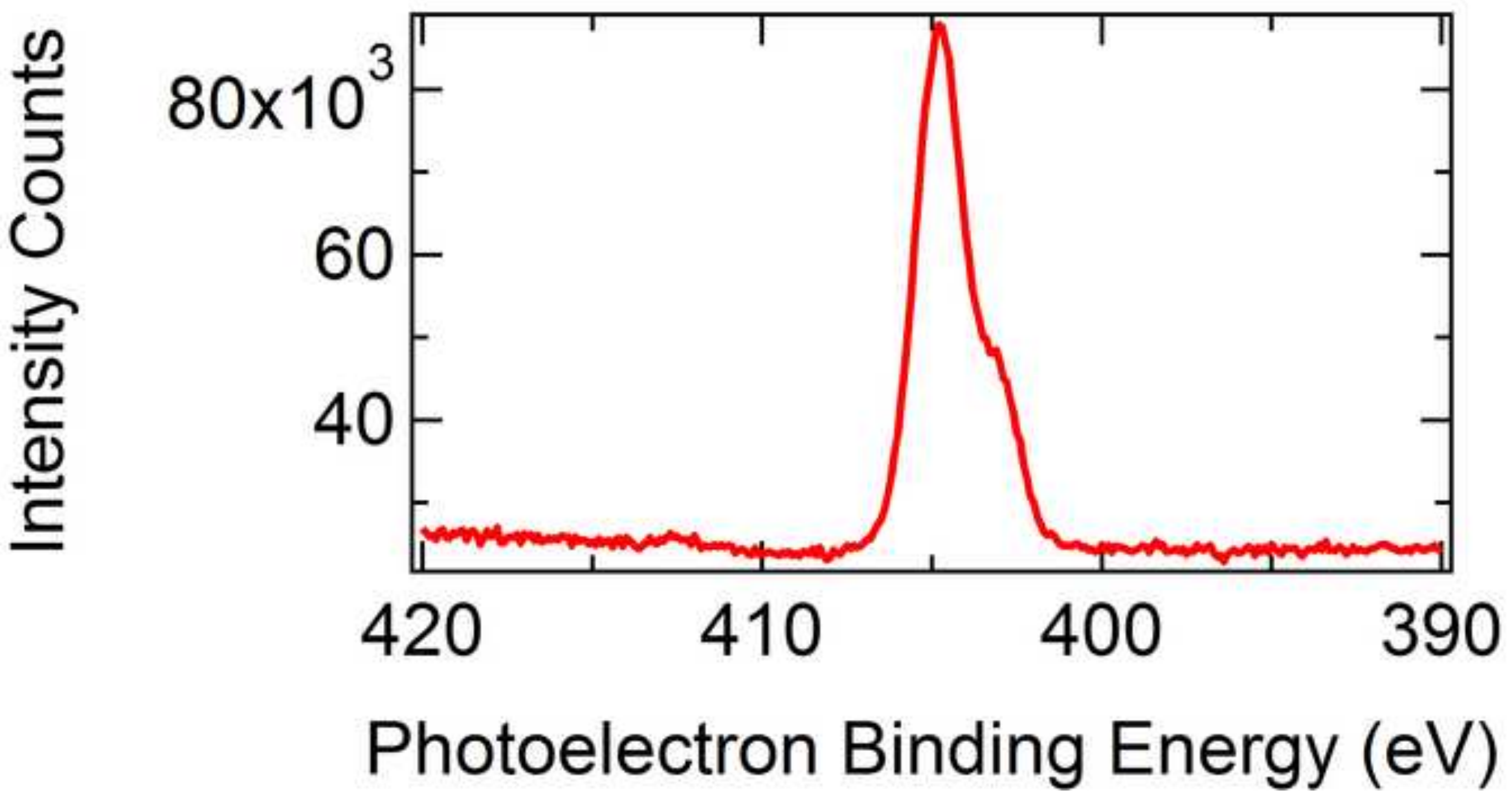




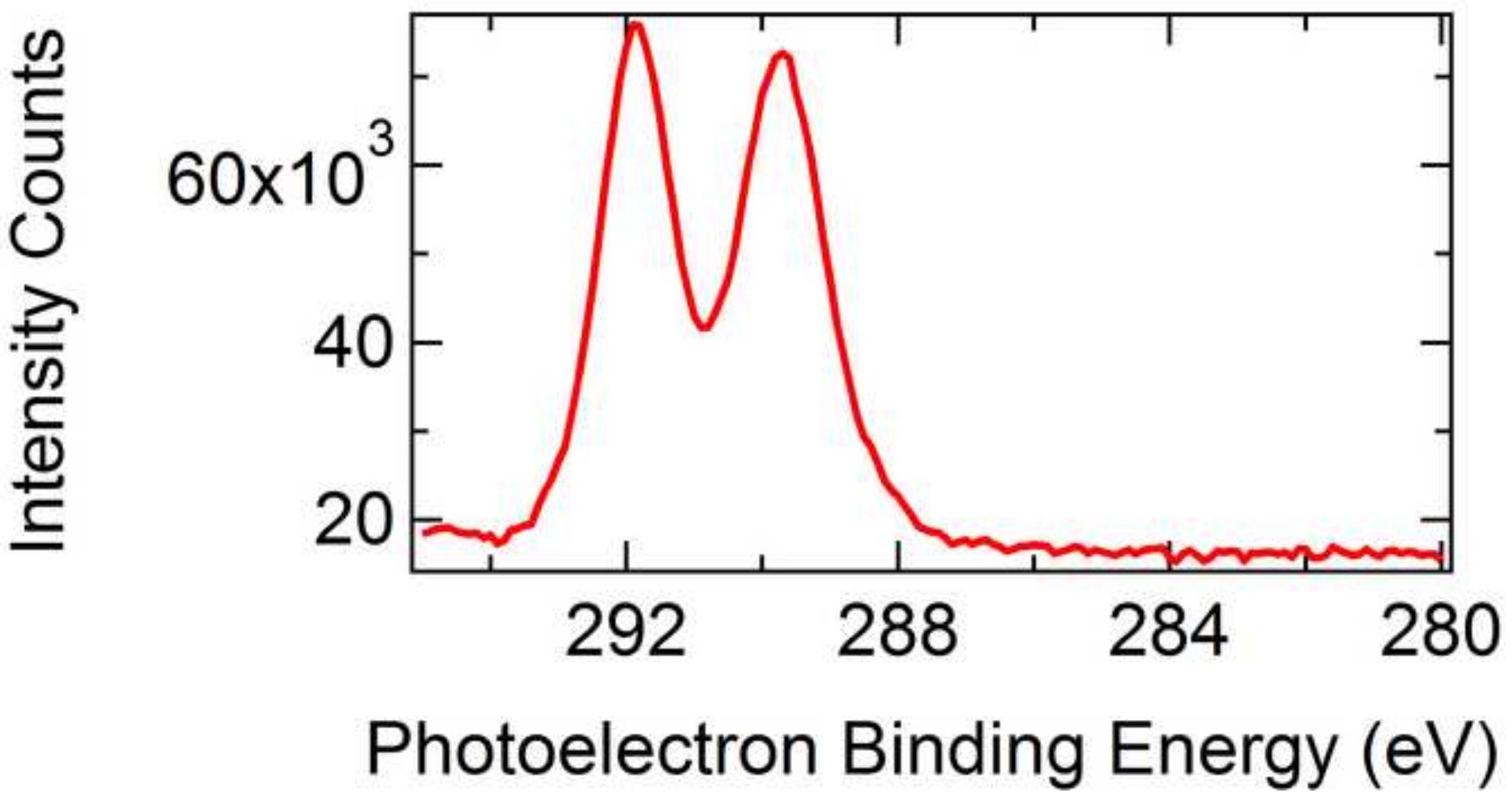


ग)

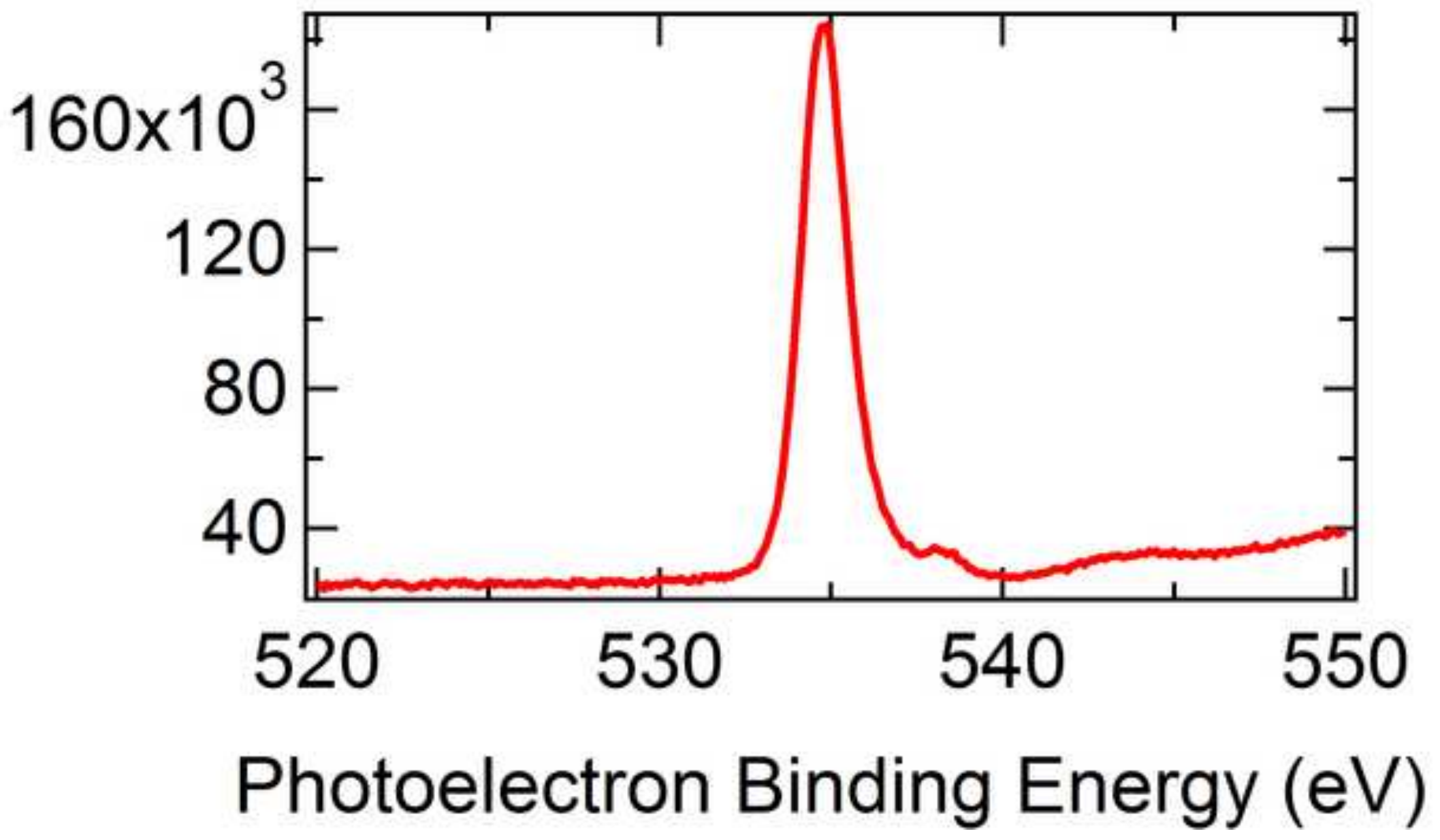




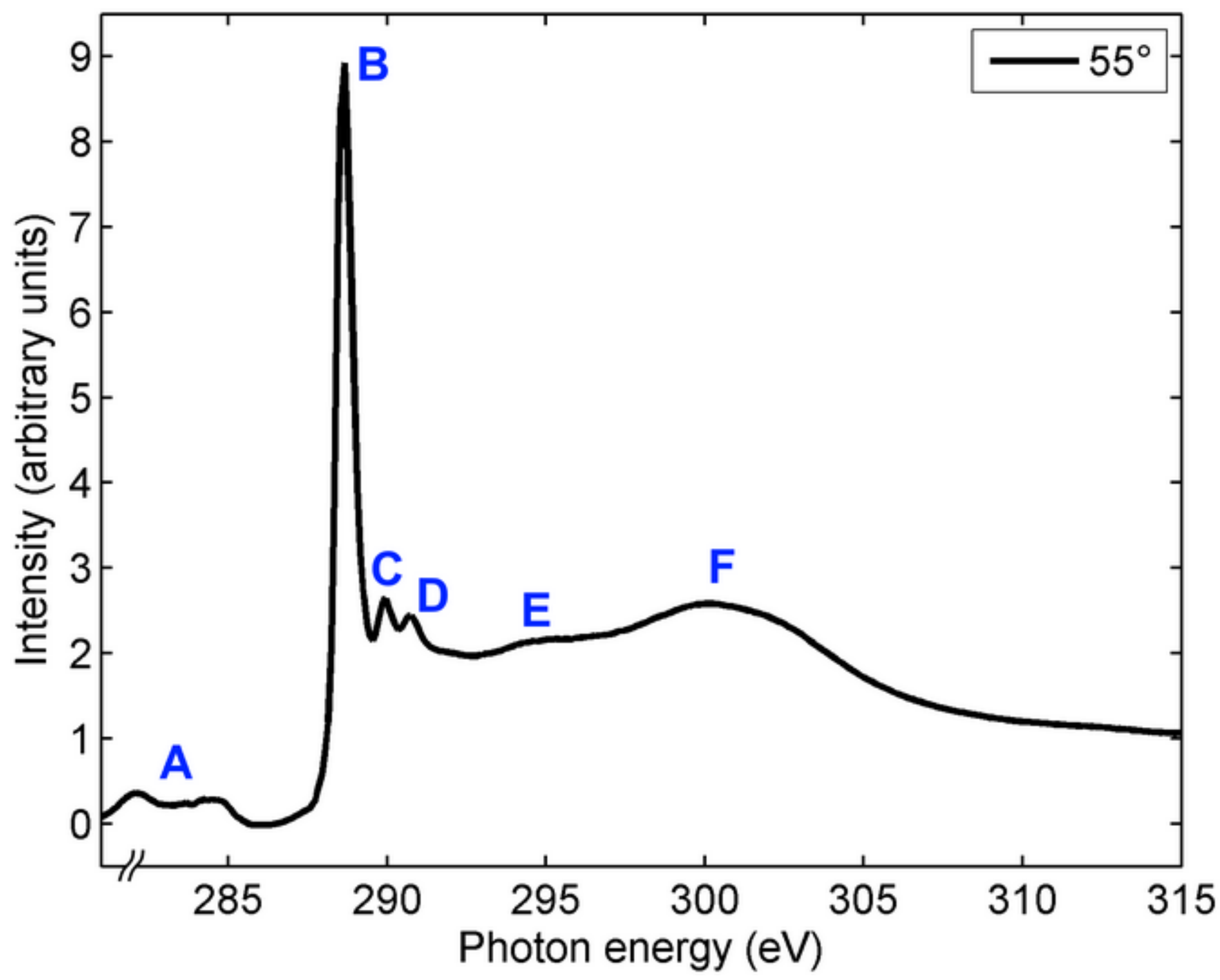



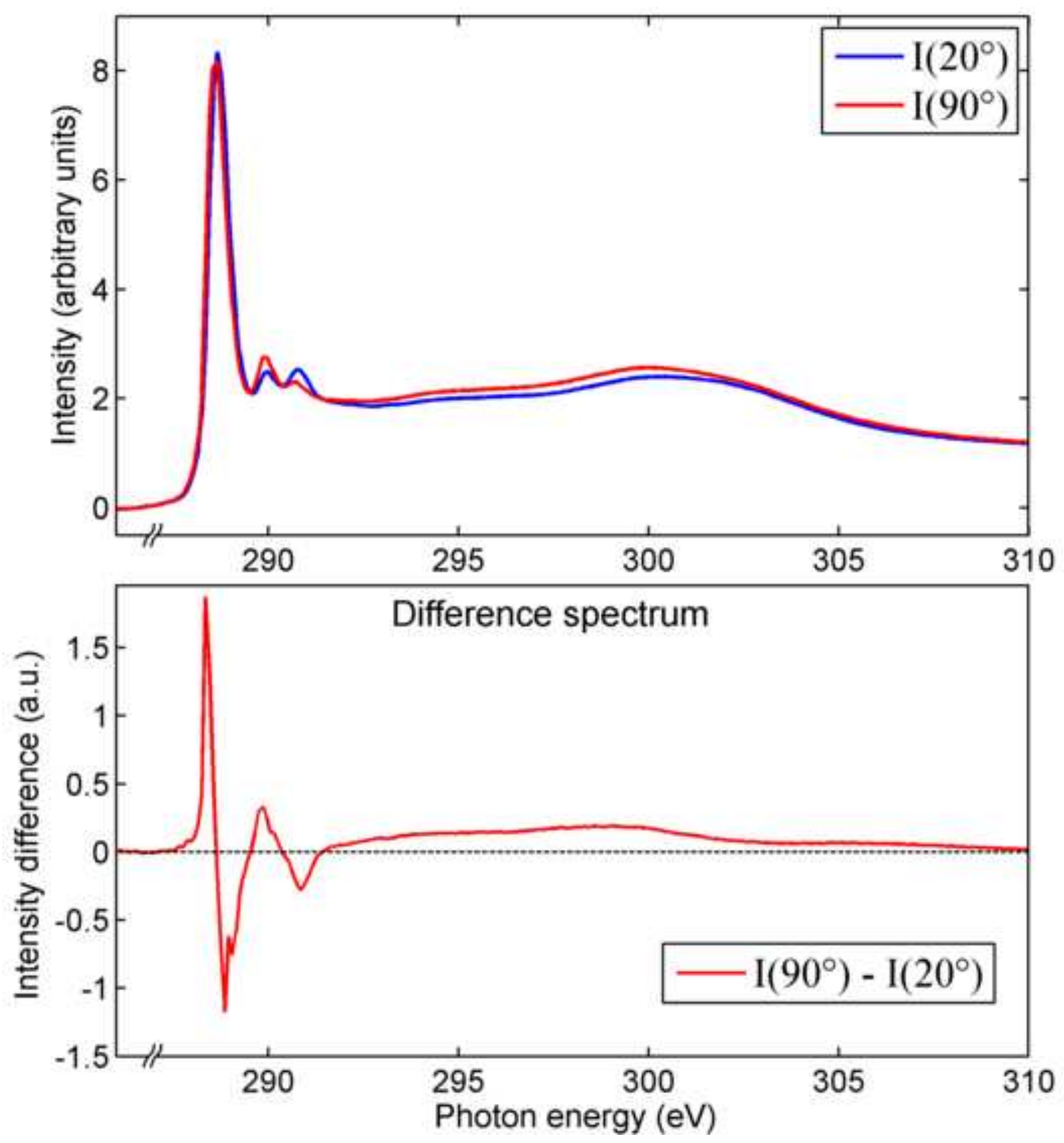